\title{
Experimental analysis and modelling of dynamic deformation and failure behaviour of steel
}

\author{
Chongyang Zeng*, Xiangfan Fang \\ Institute of Automotive Lightweight Design, University of Siegen, 57076 Siegen, Germany
}

\begin{abstract}
New specimen geometries with various stress states are designed and applied for dynamic loading tests. Oscillation-free force is measured during multiaxial loading tests in the strain range of $10^{-4}-10^{3} \mathrm{~s}^{-1}$. The deformation and local strain fields of specimens have been measured using high-speed camera and evaluated by digital image correlation (DIC) techniques. It is found that the strain rate effects on fracture strain are stressstate dependent. To model the material plasticity and ductile fracture behaviour during dynamic tests, an extended damage mechanics model (eMBW model) is used. In this work, the model is enhanced and implemented into LS-DYNA. To cover the strain rate effects on plasticity at a large strain rate range, a modified Johnson-Cook-type rate-dependency and exponential temperature-dependency are used. In addition, the influences of both stress state and strain rate on fracture locus are considered. The enhanced damage mechanics model successfully predicts the deformation and fracture behaviour of the investigated steel under dynamic multiaxial loading.
\end{abstract}

\section{Introduction}

Lightweight construction plays an important role in automotive development and environment protection. More and more lightweight metallic materials, especially high strength steels and advanced high strength steels, come to application in automotive structural components. Large multiaxial deformation and fracture might occur in the strain rate range of quasi-static (QS) to $10^{3} \mathrm{~s}^{-1}$ in vehicle crash event. Hence, accurate characterization of material deformation and fracture behaviour under various stress states, strain rates, and temperatures is necessary to improve the predictive capability of crash simulation.

\footnotetext{
* Corresponding author: chongyang.zeng@uni-siegen.de
} 
A servo-hydraulic machine is often applied to determine material dynamic properties at strain rates lower than $10^{3} \mathrm{~s}^{-1}$. However, the accurate measurement of the force signal on the servo-hydraulic machine under high-speed loading is always problematic due to the system ringing effects [1-3]. A new specimen geometry and a corresponding high-speed testing approach have been developed for oscillation-free force measurement during tensile tests in the strain range of $10^{-4}-10^{3} \mathrm{~s}^{-1}$ in previous studies [4-5].

For automotive crash simulation with finite element method (FEM), dynamic constitutive models related to strain rate and adiabatic heating are essential for correctly modelling material plasticity. Different phenomenological constitutive models have been proposed over the past few decades [6-9]. To model material ductile fracture behaviour, many fractures models like Johnson-Cook (J-C) [6], CrachFEM [10], GISSMO [11], Bai-Wierzbicki [1213] and Hosford-Coulomb [8] are proposed. A hybrid damage mechanics model (namely MBW model), which introduces a damage initiation concept and damage induced softening is developed by Lian et al. [14] based on the fracture model proposed by Bai and Wierzbicki [12]. The model is subsequently enhanced through introducing different effects, such as the strain rate, temperature effects [9] and loading history effects [15]. The MBW model is further developed as a comprehensive and concise model, which is referred to as the extended MBW (eMBW) model [16].

In this study, different fracture specimens (shear, central hole, notched and plane-strain) are designed for multiaxial dynamic tensile tests at strain rate up to $10^{3} \mathrm{~s}^{-1}$. Tensile tests of a micro-alloy HX340LAD are performed under different strain rates and temperatures. The influences of stress state and strain rate on material deformation and failure behaviour are also investigated. The eMBW model is extended by including a modified J-C-type ratedependency and a strain rate dependent fracture initiation locus. The enhanced model is implemented into LS-DYNA and validated through a comparison of the experimental and simulated force-displacement response.

\section{Experiments}

\subsection{Material and specimens}

The investigated material is a $1.5 \mathrm{~mm}$ thick micro-alloyed steel (HX340LAD) sheet. Since the influence of strain rate on damage/fracture behaviour might vary for different stress states, high-speed multiaxial tensile tests are necessary to study the influence of strain rate on the damage/fracture locus.

Fig. 1 (a) shows the designed specimens with different stress states. For the design of fracture gauge section, shear specimen ( $\mathrm{SH}$ ) is designed with two symmetrical local notches based on the previous study by Peirs, et al. [17]. The central hole specimen (CH-R3) has a hole diameter of $6 \mathrm{~mm}$ and a $16 \mathrm{~mm}$ wide-gauge section. A notched dog-bone specimen (NDB-R8) is designed with two symmetrical notches. The notch radius is $8 \mathrm{~mm}$ and the minimum gauge section is $6 \mathrm{~mm}$. The plane-strain specimen (PS-R4) includes two symmetrical notches with a radius of $4 \mathrm{~mm}$, which are manufactured through the thickness direction of the specimen. The minimum thickness in notches is $0.9 \mathrm{~mm}$. A more systematic investigation of the effects of radius on the stress states in geometry design is found in [18].

Based on the proposed stress wave model by Fang [5], the force-displacement response can also be obtained for the high-speed multiaxial tensile tests without ringing effects. As shown in Fig. 1 (a), for each specimen a second specimen shoulder (elastic measurement area: EMA) and a unique minor plastic deformation area (MinDA) are designed for the oscillation-free force measurement under dynamic loading condition. The clamping system of high-speed machine with the GEN III specimen is shown in Fig. 1 (b). During tension, a 
homogenous stress/strain distribution in EMA of specimens can be obtained due to the special design of MinDA. The actual sizes of MinDA have been optimized separately for different specimens through the FE simulation with LS-DYNA according to the principle of GEN III specimens [4-5]. Fig. 2 shows the simulative strain distribution pattern of EMA for different specimens before the onset of fracture. The strain pattern in EMA stays mostly the same throughout the entire loading history. The black solid square on the second shoulder shows the position where a strain gauge should be attached for the force measurement under dynamic loading.

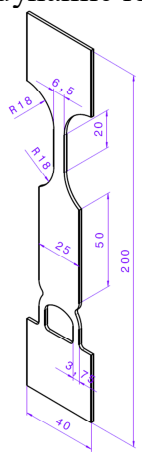

GEN III (SDB )

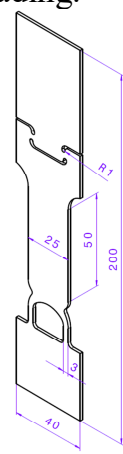

$\mathrm{SH}$

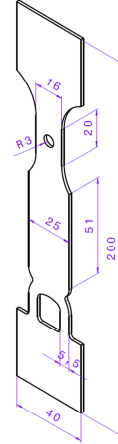

CH-R3

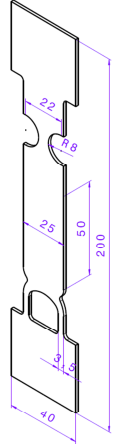

NDB-R8

(a)

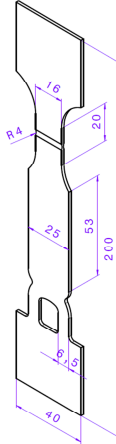

PS-R4

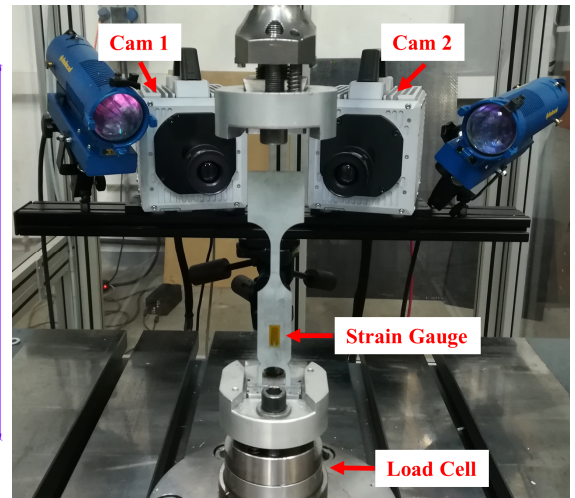

(b)

Fig. 1. (a) Overview of the geometry of plasticity and fracture specimens (mm); (b) Experimental setup with servo-hydraulic machine and high-speed cameras.

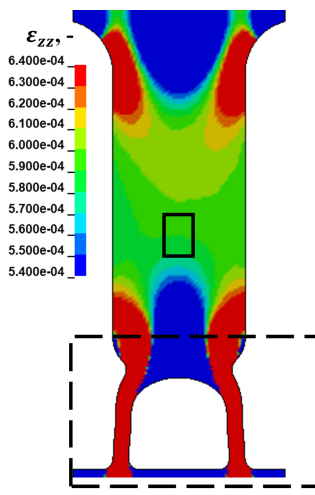

$\mathrm{SH}$

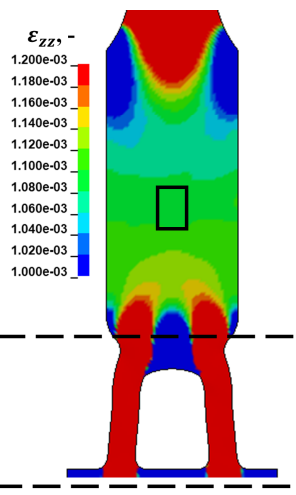

CH-R3

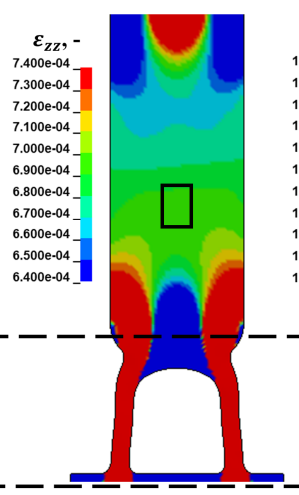

NDB-R8

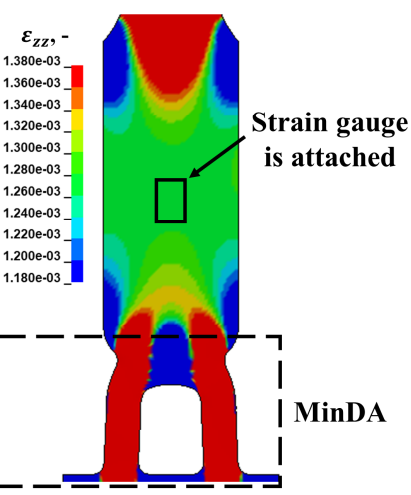

PS-R4

Fig. 2. Strain distribution pattern of different specimens before the onset of fracture.

The force versus time curves of tensile tests with loading speeds of $2 \mathrm{~m} / \mathrm{s}$ and $10 \mathrm{~m} / \mathrm{s}$ measured by the load cell and strain gauge (see Fig. 1 (b)) are shown in Fig. 3. It is found that the quality of the force signal by strain gauge is much higher than the data measured by the load cell. The geometries of fracture specimens are validated with the experimental data. With the almost ringing-free force-displacement response, model parameters related to material plasticity or damage/fracture can be calibrated more accurately.

All specimens are manufactured by CNC milling to obtain good surface condition and accurate size. The tensile specimens are manufactured along material rolling direction. 


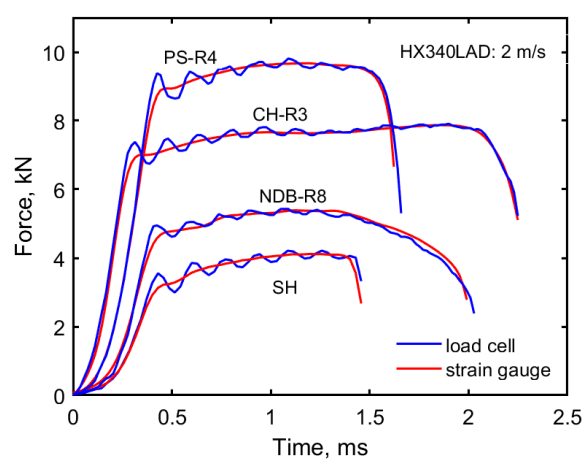

(a)

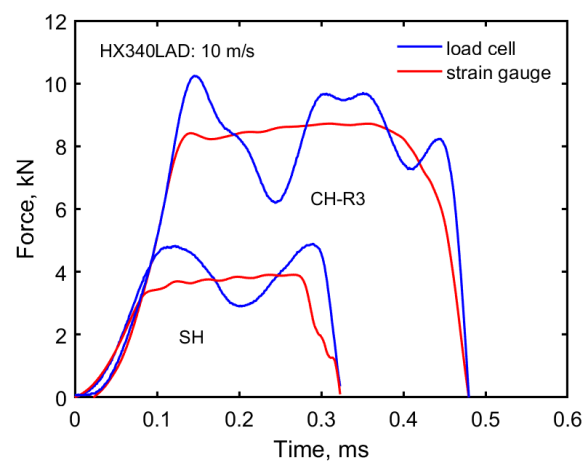

(b)

Fig. 3. Experimental force signal measured by the load cell and strain gauge. (a) $2 \mathrm{~m} / \mathrm{s}$; (b) $10 \mathrm{~m} / \mathrm{s}$.

\subsection{Experimental techniques}

For low strain rates $\left(\leq 0.1 \mathrm{~s}^{-1}\right)$ and low loading velocities $(<0.5 \mathrm{~mm} / \mathrm{s})$, the tensile tests are executed on a $100 \mathrm{kN}$ testing machine (Zwick Z100). The force is directly measured by the load cell. The displacement, strain field and local strain at fracture are measured using DIC method. Two Aramis 5M CCD cameras with maximum frame rate of $29 \mathrm{~Hz}$ are applied.

For dynamic tensile tests, the servo-hydraulic tensile machine HTM 5020 (see Fig. 1 (b)) is employed. The maximum loading velocity of HTM 5020 is $20 \mathrm{~m} / \mathrm{s}$, which corresponds to a nominal strain rate of $10^{3} \mathrm{~s}^{-1}$ with the GEN III specimen. The force is measured in the EMA of specimen shoulder with strain gauges, as shown in Fig.1 (b). The force is calculated according to Eq. (1),

$$
F=\varepsilon_{z z} \cdot E \cdot A
$$

where $\varepsilon_{z z}$ is the elastic strain in the tensile direction; $\mathrm{E}$ is Young's modulus; and $\mathrm{A}$ is the cross-section of the specimen in the area where strain gauge sensor measurements take place. Two SA5 PHOTRON high-speed cameras (maximum frame rate: $1 \mathrm{MHz}$ ) are used to record the pictures during specimen deformation. A more detailed setup of high-speed tests is found in [4].

\subsection{Experimental procedures}

In this work, an experimental program considering material plasticity and fracture behaviour under different strain rates and temperatures is carried out, which is listed in Table 1. For all different loading cases, three parallel tests have been conducted.

Table 1. Experimental program under different strain rates and temperatures.

\begin{tabular}{|c|c|c|c|c|c|}
\hline \multirow[t]{2}{*}{ Uniaxial tension at RT } & \multicolumn{5}{|c|}{ Strain rate, $\mathrm{s}^{-1}$} \\
\hline & 0.0005 & 0.01 & 0.1 & 20 & 1000 \\
\hline \multirow[t]{2}{*}{ Uniaxial tension at QS } & \multicolumn{5}{|c|}{ Temperature, ${ }^{\circ} \mathrm{C}$} \\
\hline & -30 & & 20 & & 100 \\
\hline \multirow{2}{*}{$\begin{array}{c}\text { Fracture tests } \\
\text { (SH, CH-R3, NDB-R8, } \\
\text { PS-R4) }\end{array}$} & \multicolumn{5}{|c|}{ Loading speed, $\mathrm{m} / \mathrm{s}$} \\
\hline & $5 \times 10^{-6}$ & $5 \times 10^{-4}$ & 0.2 & 2 & 10 \\
\hline
\end{tabular}




\section{Experimental results analysis and parameters calibration}

\subsection{Damage mechanics model (eMBW)}

The yield function of the eMBW model [16] is defined according to Eq. (2),

$$
\Phi=\bar{\sigma}-(1-D) \cdot \sigma_{y}\left(\bar{\varepsilon}^{P}\right) \cdot f\left(\dot{\bar{\varepsilon}}_{p}\right) \cdot f(T) \cdot f(\bar{\theta}) \leq 0
$$

where $\bar{\sigma}$ is the equivalent stress, $\mathrm{D}$ is the damage variable, $\sigma_{y}\left(\bar{\varepsilon}^{P}\right)$ is the flow curve at QS and room temperature (RT) condition; $f\left(\dot{\bar{\varepsilon}}_{p}\right), f(T)$ and $f(\bar{\theta})$ are the effects of strain rate, temperature and lode angle parameter on material plasticity, respectively.

The ductile damage initiation and fracture strain are defined by Eq. (3) and Eq. (4), respectively. If the stress triaxiality is lower than $\eta_{c}$, damage or fracture do not occur.

$$
\begin{gathered}
\bar{\varepsilon}_{d d i}^{P}\left(\eta_{\text {avg },} \bar{\theta}_{\text {avg }}\right)= \begin{cases}+\infty ; & \eta \leq \eta_{c} \\
{\left[D_{1} e^{-D_{2} \eta}-D_{3} e^{-D_{4} \eta}\right] \bar{\theta}^{2}+D_{3} e^{-D_{4} \eta} ;} & \eta \geq \eta_{c}\end{cases} \\
\bar{\varepsilon}_{d f}^{P}\left(\eta_{\text {avg, }} \bar{\theta}_{\text {avg }}\right)= \begin{cases}+\infty ; & \eta \leq \eta_{c} \\
{\left[F_{1} e^{F \eta}-F_{3} e^{-F_{4} \eta}\right] \bar{\theta}^{2}+F_{3} e^{F \eta} ;} & \eta \geq \eta_{c}\end{cases}
\end{gathered}
$$

In this study, the damage initiation is identical to the fracture initiation. That means the damage initiation is set as the same with fracture strain. The effects of the Lode angle parameter on plasticity are not considered in this study. More model description and parameters definition are found in [16].

\subsection{Flow curve identification at RT and QS}

GEN III specimen (Fig. 1 (a)) with gauge length of $20 \mathrm{~mm}$ is used to determine the material mechanical properties. The true stress-true plastic strain curve until ultimate tensile strength (UTS) obtained at RT and QS for material HX340LAD is shown in Fig. 4. Only one curve is shown because of the excellent repeatability of the three parallel experiments. In the simulation, the flow curve is needed with a large range of strain. Hence, a combined SwiftVoce law (see Eq. (2)) is used for the flow curve derivation based on RT and QS tensile test results. The fitting result is also plotted in Fig. 4. The detailed calibration procedures are found in [18].

$$
\bar{\sigma}_{y}^{S-V}\left(\bar{\varepsilon}^{P}\right)=\alpha \cdot A\left(\bar{\varepsilon}_{0}+\bar{\varepsilon}^{P}\right)^{n}+(1-\alpha) \cdot\left[k_{0}+Q\left(1-e^{-\beta \bar{\varepsilon}^{P}}\right]\right.
$$

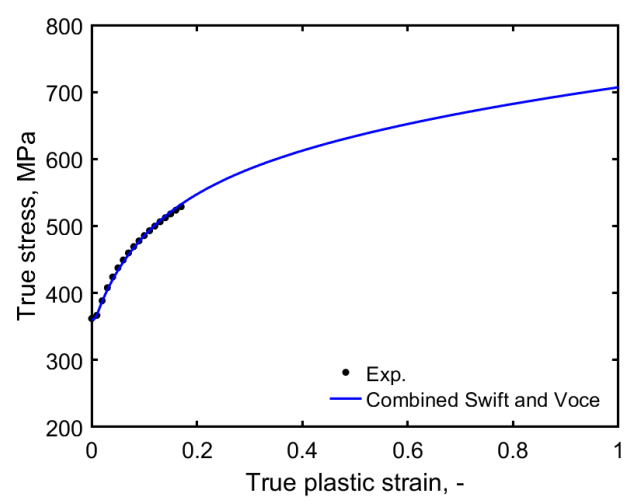

Fig. 4. Experimental and the extrapolated flow curves according to the combined Swift-Voce law. 


\subsection{Temperature effects}

Fig. 5 (a) shows the true stress-true plastic strain curves until the corresponding UTS obtained at QS under different temperatures. The stresses at the true plastic strain of 0.02 are used to evaluate the temperature sensitivity. Since adiabatic heating is not taken into account in QS tests, the temperature sensitivity is calibrated directly based on this data. An exponential temperature effect term $f(T)$ (see Eq. (2)) is used for temperature sensitivity calibration. The fitting result for temperature dependence is shown in Fig. 5 (b).

$$
f(T)=c_{1}^{T} \cdot \exp \left(c_{2}^{T} \cdot T\right)+c_{3}^{T}
$$

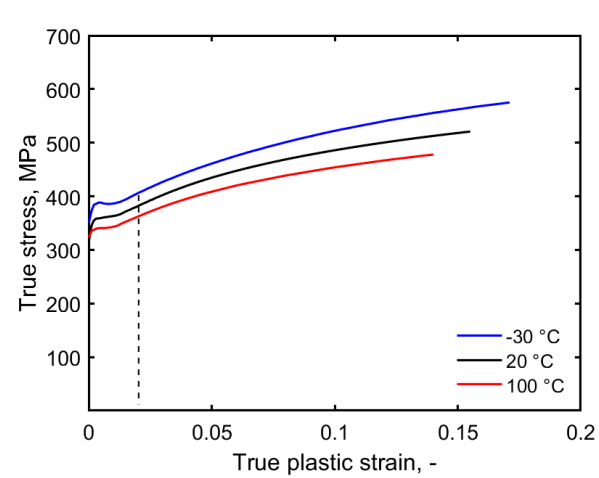

(a)

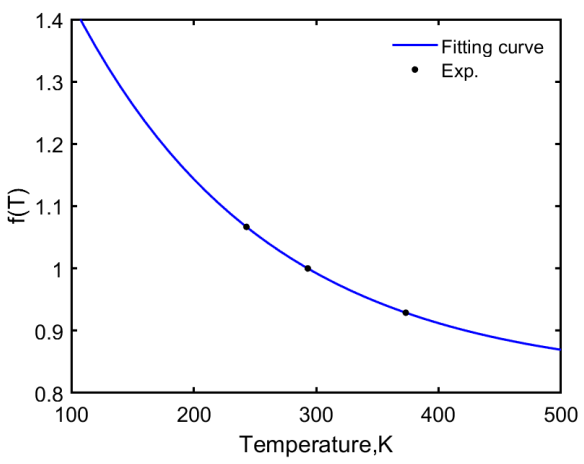

(b)

Fig. 5. (a) True stress - true plastic strain curves for various temperatures; (b) Temperature dependence fitting.

\subsection{Strain rate effects}

To calculate the strain rate effects, the isothermal stress-strain response is corrected according to Eq. (7). The instantaneous $f(T)$ is calculated with the temperature increase $\Delta T$ during plastic deformation. The $\Delta T$ is obtained based on the conservation of energy from plastic work to heat by assuming a certain fraction of the plastic work is released to the environment. Taylor-Quinney coefficient $\beta$ is used here to describe the transition from isothermal to adiabatic condition, as shown in Eq. (8). The procedure for conducting the correction and parameters fitting is proposed in detail in previous research [9]. Both material constants and $\beta$ are assumed as constant during the plastic deformation. The experimental adiabatic and calculated isothermal true stress-true plastic strain curves until the corresponding UTS for different strain rates are shown in Fig. 6 (a). The Taylor-Quinney coefficient $\beta$ used in this study is plotted in Fig. 6 (b).

$$
\begin{gathered}
\sigma_{i s o}=\frac{\sigma_{a d i}}{f(T)} \\
\Delta T=\frac{\beta}{\rho \cdot C_{p}} \int_{0}^{\varepsilon} \sigma_{i s o} d \varepsilon
\end{gathered}
$$

For the strain rate sensitivity parameter calibration, the true stress-true plastic strain response at RT and QS is assumed as reference. The stresses at the true plastic strain of 0.02 are used to evaluate the strain rate effects. Fig. 7 (a) shows the experimental data and the strain rate dependence fitting. The black points are the average experimental data. The red curve shows the fitting results of strain rate dependence with original J-C-type strain rate term (see Eq. (9)) by assuming the tensile results are only available under low strain rates. 
The blue curve shows the fitting results with a modified J-C strain rate term, which is shown in Eq. (10).

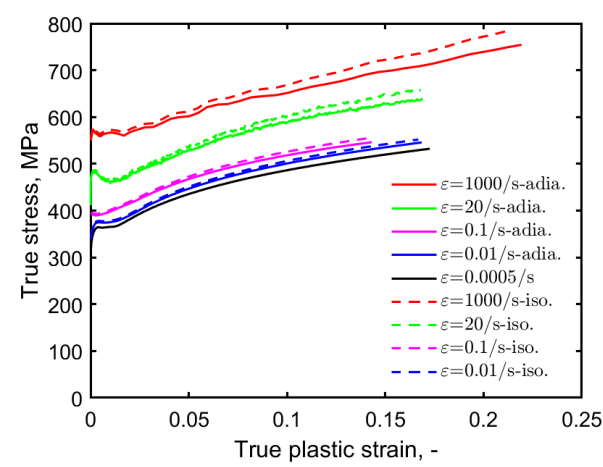

(a)

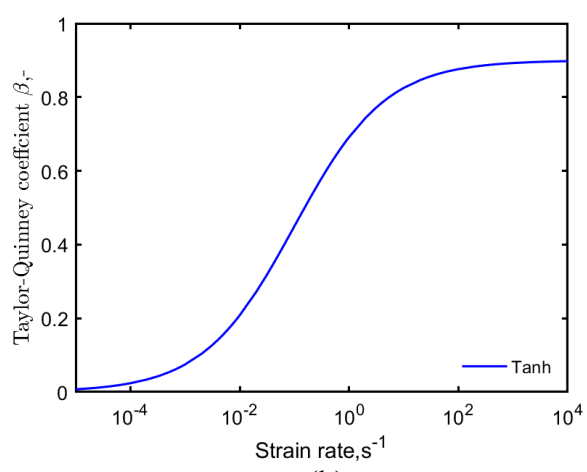

(b)

Fig. 6. (a) Adiabatic and isothermal flow curves at different strain rates; (b) The Taylor-Quinney coefficient $\beta$ as the function of strain rate.

It can be easily recognized that the original J-C-type strain rate term with one parameter cannot adequately describe the strain rate sensitivity in dynamic region. A nonlinear relationship between $f(\dot{\varepsilon})$ and strain rate is found according to the experimental data. The modified J-C strain rate term fit quite well with the normalized stress during strain rate evolution in the strain rate range of $10^{-4}-10^{3} \mathrm{~s}^{-1}$. The values of parameters $\mathrm{a}, \mathrm{b}$ and $\mathrm{c}$ versus strain are plotted in Fig. 7 (b).

$$
\begin{gathered}
f(\dot{\varepsilon})=1+C_{1} \cdot \ln \left(\frac{\dot{\bar{\varepsilon}}_{p}}{\dot{\varepsilon}_{0}}\right) \\
f(\dot{\varepsilon})=1+a \cdot \ln \left(\frac{\dot{\varepsilon}_{p}}{\dot{\varepsilon}_{0}}\right)+b \cdot\left[\ln \left(\frac{\dot{\varepsilon}_{p}}{\dot{\varepsilon}_{0}}\right)\right]^{2}+c \cdot\left[\ln \left(\frac{\dot{\varepsilon}_{p}}{\dot{\varepsilon}_{0}}\right)\right]^{3}
\end{gathered}
$$

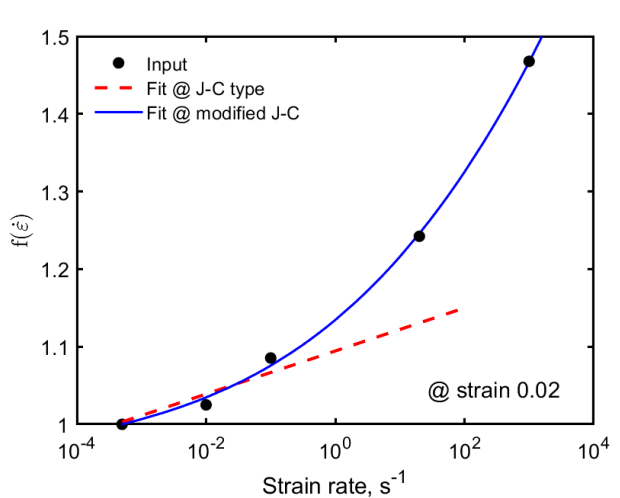

(a)

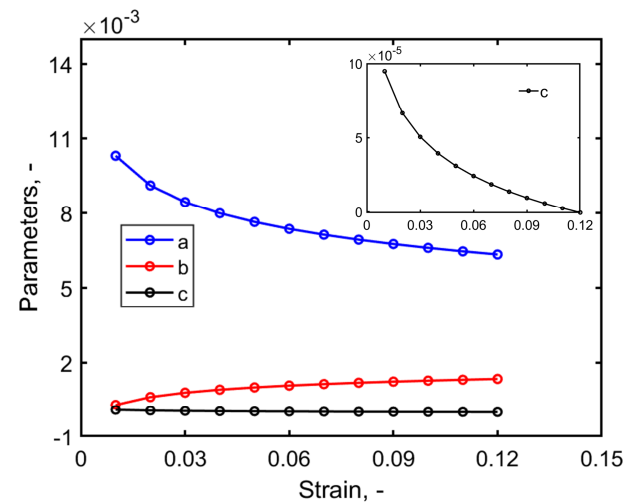

(b)

Fig. 7. (a) Strain rate dependence fitting with original J-C and modified J-C strain rate term; (b) Parameters $\mathrm{a}, \mathrm{b}$ and $\mathrm{c}$ for different strains

\subsection{Fracture characterization}

The force-displacement curves for SH, CH-R3, NDB-R8 and PS-R4 tensile tests under various loading speeds are shown in Fig. 8. A gauge length of $10 \mathrm{~mm}$ is used to evaluate the displacement. It is found that with increasing loading speed, the force level exhibits a significantly increasing trend, especially at the beginning. For SH and PS-R4 specimens, 
shorter fracture displacements are observed with increasing loading speeds in dynamic region. While the displacement up to fracture for $\mathrm{CH}-\mathrm{R} 3$ and NDB-R8 specimens seems to get less influence from the loading speed.

Local fracture strain is determined with DIC as the equivalent strain before the appearance of the first visible crack on the specimen surface. The sudden decrease of force is an additional way to locate the crack initiation. In this work, the crack initiation (local fracture strain) is determined by combining both methods. A uniform reference length of $0.2 \mathrm{~mm}$ is used for local strain evaluation. Fig. 9 (a) shows the surface fracture strain versus strain rate for different specimen geometries. The strain rates under different loading speeds are the average real strain rates, which are evaluated from the crack initiation point. A normalized fracture strain is plotted as the function of $\ln \left(\frac{\dot{\bar{\varepsilon}}_{p}}{\dot{\varepsilon}_{0}}\right)$, which is shown in Fig. 9 (b). It is found that for NDB-R8 specimen the strain rate has a slight positive effect on fracture strain. While for SH and PS-R4 specimens the fracture strain decreases significantly with increasing strain rates (loading speeds).
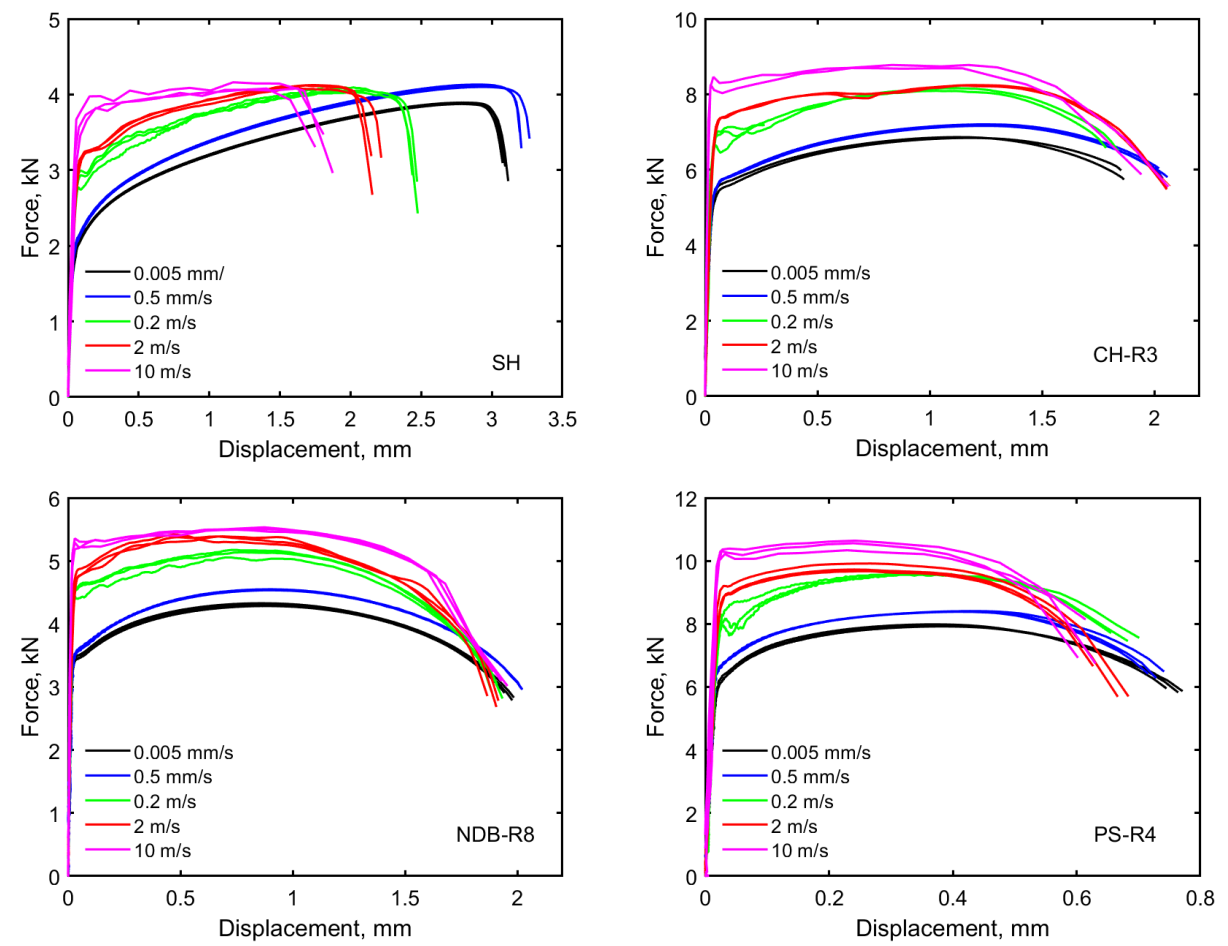

Fig. 8. Force-displacement curves of HX340LAD under different loading speeds for four different specimen geometries. 


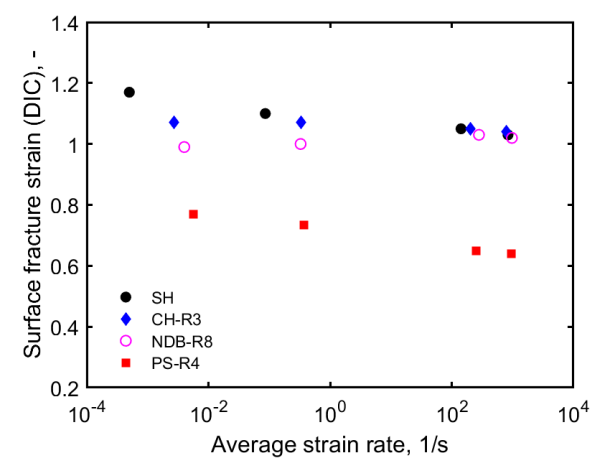

(a)

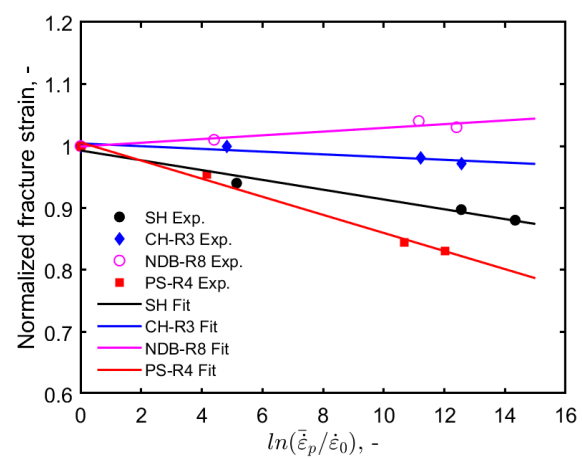

(b)

Fig. 9. (a) Surface fracture strain measured by DIC; (b) Strain rate effect on fracture strain for different specimen geometries.

\section{Numerical simulation}

In this work, FE simulations are carried out using LS-DYNA explicit solver. A solid element with size of $0.1 \mathrm{~mm} \times 0.1 \mathrm{~mm} \times 0.1 \mathrm{~mm}$ in critical deformation zone is chosen. Since the full model with solid mesh size of $0.1 \mathrm{~mm}$ requires a large simulation time, only one fourth of each specimen within the gauge section is modelled.

Fracture is normally assumed to initiate from the inside of strain localization zone of specimens. Comparing central elements, surface elements show quite a smaller equivalent plastic strain in large strain range. Since the DIC can only measure the strain information on the specimen surface, the fracture initiation locus (see Fig. 10 (a)) used in this study is calibrated in FEM simulation. Based on the experimental data in Fig. 9 (b) and the previous work by Mohr, et al. [8], a lode angle dependent strain rate term is assumed and incorporated into fracture initiation locus of eMBW model (given by Eq. (4)), which is shown in Eq. (11). The effect of strain rate on fracture strain is controled though the coefficient $\chi(\bar{\theta})$, which is plotted in Fig. 10 (b). The $\chi(\bar{\theta})$ is calibrated by comparing the experimental and simulative force-displacement curves. The calibrated value of $\chi(\bar{\theta})$ has some slight differences with DIC results. Further studying of the effect of strian rate on fracture behaviour is planned for future work.

$$
\bar{\varepsilon}_{f}(\eta, \bar{\theta}, \dot{\varepsilon})=\left[1+\chi(\bar{\theta}) \cdot \ln \left(\frac{\dot{\varepsilon}_{p}}{\dot{\varepsilon}_{0}}\right)\right] \cdot\left[\left(F_{1} e^{F \eta}-F_{3} e^{-F_{4} \eta}\right) \bar{\theta}^{2}+F_{3} e^{F \eta}\right]
$$

Fig. 11 shows the comparison of experimental and simulative force-displacement curves under loading speeds of $0.005 \mathrm{~mm} / \mathrm{s}$ (QS) and $2 \mathrm{~m} / \mathrm{s}$ for two different specimen geometries. It can be easily recognized that with the proposed strain rate dependent fracture locus the predictable capability of eMBW model has been significantly improved. 


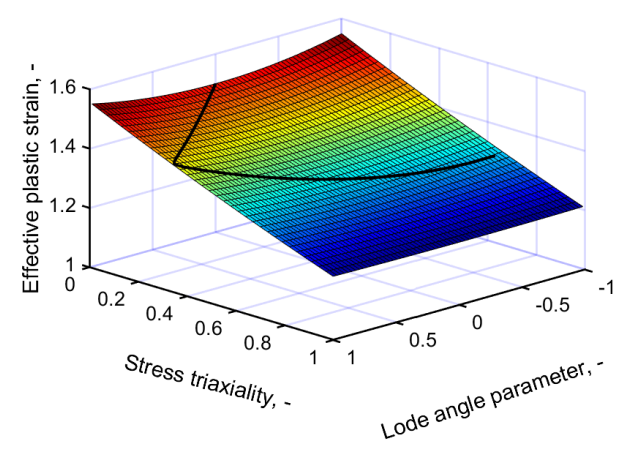

(a)

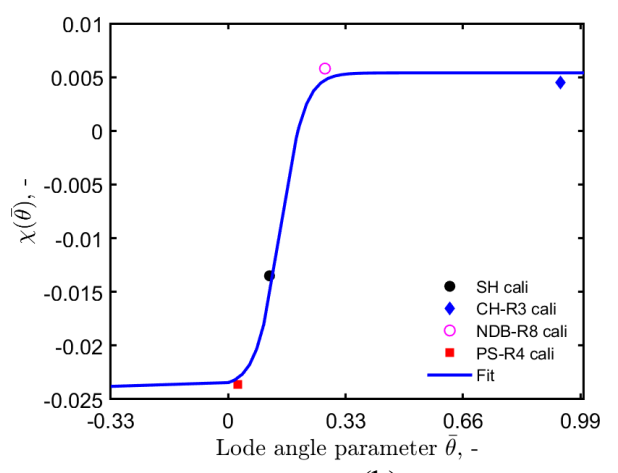

(b)

Fig. 10. (a) Calibrated fracture initiation locus at $0.005 \mathrm{~mm} / \mathrm{s}(\mathrm{QS})$, (b) Coefficient $\chi(\bar{\theta})$.

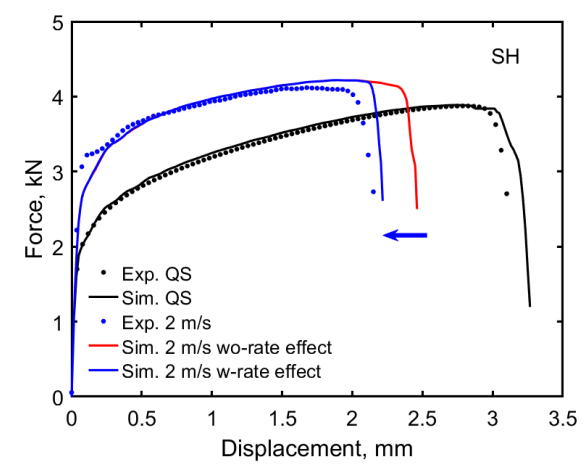

(a)

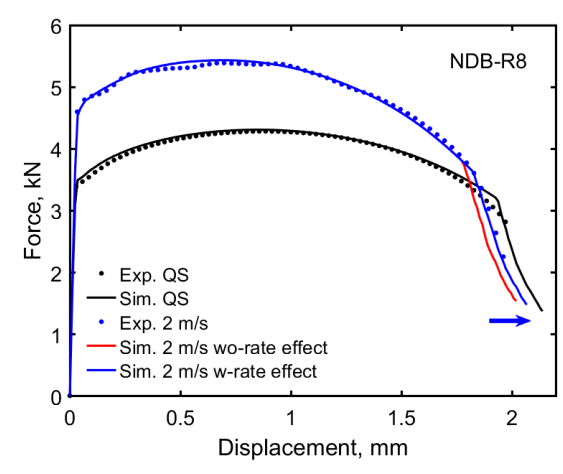

(b)

Fig. 11. Force-displacement curves from experiments and the simulations at different loading speeds for different geometries. (a) SH; (b) NDB-R8

\section{Conclusions}

In this study, the fracture specimens are designed and performed for multiaxial tensile tests at the strain rate from QS to $10^{3} \mathrm{~s}^{-1}$. The effects of strain rate and temperature on deformation and fracture initiation of HX340LAD are investigated. Accurate characterization of strain rate sensitivity under dynamic loading is realized with the modified J-C strain rate term.

For specimens with low Lode angle parameters (SH and PS-R4) the strain rate has a significant negative effect on fracture strain. While for CH-R8 and NDB-R3, which normally have a relative larger Lade angle parameter, the effect of strain rate on fracture strain is slightly positive.

A strain rate-dependent fracture initiation locus is proposed which couples with eMBW model and successfully predicts the onset of fracture in static and dynamic multiaxial experiments.

The support through the German Research Foundation (Deutsche Forschungsgemeinschaft, grant No.: FA 1024/5-1) is gratefully acknowledged. Special thanks are due to Prof. Sebastian Münstermann for original eMBW subroutine support. The authors are also grateful for valuable discussions from Prof. Junhe Lian. 


\section{References}

1. X. Xiao, In Proc. of the XIth International Congress and Exposition, Orlando, Florida, USA (2008)

2. D. Zhu, S. D. Rajan, B. Mobasher, A. Peled, M. Mignolet, Exp. Mech., 51(8), 13471363 (2011)

3. J. Li, X. F. Fang, Exp. Mech., 54(8), 1497-1501 (2014)

4. X. F. Fang, R. Grams, JTE, 49(3) (2019)

5. X. F. Fang, Int. J. Impact Eng., 149, 103770 (2021)

6. G. R. Johnson, W. H. Cook, Eng. Fract. Mech., 21(1), 31-48 (1985)

7. A. Rusinek, J R. Klepaczko, Int. J. Plast., 17(1), 87-115 (2001)

8. C. C. Roth, D. Mohr, Int. J. Plast., 56, 19-44 (2014)

9. D. Novokshanov, B. Döbereiner, M. Sharaf, S. Münstermann, J. Lian, Eng. Fract. Mech., 148, 281-303 (2015)

10. H. Gese, G. Oberhofer, H. Dell, Dyna 6 (2007)

11. F. Neukamm, M. Feucht, A. Haufe, LS-Dyna Anwenderforum, 30, 11-20 (2008)

12. Y. Bai, T. Wierzbicki, Int. J. Plast., 24(6), 1071-1096 (2008)

13. T. Wierzbicki, Y. Bai, Int J Fract, 161, 1-20 (2010)

14. J. Lian, M. Sharaf, F. Archie, S. Münstermann, Int. J. Damage Mech., 22, 188-218 (2013)

15. B. Wu, X. Li, Y. Di, V. Brinnel, J. Lian, S Münstermann, Fatigue Fract Eng Mater Struct, 40(12), 2152-2168 (2017)

16. W. Liu, J. Lian, S. Münstermann, C. Zeng, X. F. Fang, Int. J. Mech. Sci., 176, 105534 (2020)

17. J. Peirs, P. Verleysen, J. Degrieck, Exp. Mech. 52(7), 729-741 (2012)

18. W. Liu, J. Lian, S. Münstermann, Eng. Fail. Anal., 106, 104138 (2019) 